\title{
Quantal cumulant dynamics II. An efficient time-reversible integrator
}

\author{
Yasuteru Shigeta, ${ }^{* a}$, Hideaki Miyachi ${ }^{\mathrm{b}}$, Kimihiko Hirao $^{\mathrm{b}}$ \\ ${ }^{a}$ Department of Physics, Graduate School of Pure and Applied Science, University \\ of Tsukuba, Tennodai 1-1-1, Ibaraki 305-8571, Japan \\ b Department of Applied Chemistry, Graduate School of Engineering, The \\ University of Tokyo, 7-3-1 Hongo, Bunkyo-ku, Tokyo, 113-8656, Japan
}

\begin{abstract}
The applicability of time-reversible integrators to the recently developed quantal cumulant dynamics (QCD) is examined, and their accuracy and efficiency are demonstrated by comparison with the Runge-Kutta method. We proposed three schemes, which differ in their partitions and orders of an exponential function of a Liouvillian. Three-part partition conserves the total energy with sufficient accuracy, whereas the two-part one does not. It is found that the equations of motion of the QCD are sensitive to the order. The sensitivity is due to the accordance of fractional time in variables, which contribute to the total energy, after the propagation.
\end{abstract}

* Corresponding author. FAX: +81 298535924.

Email address: shigeta@comas.frsc.tsukuba.ac.jp (Yasuteru Shigeta,). 


\section{Introduction}

Quantum dynamics simulations are powerful tools for investigating the time evolution of physical quantities and hence enhancing our understanding of dynamical properties of quantum many-body systems. Nevertheless, the manybody dynamics of chemical reactions are too complicated and demanding for a simulation using the methods of full quantum dynamics. Therefore, the full quantum dynamics method is limited to several degrees of freedom. Instead of quantum dynamics, classical dynamics has been used routinely for analyzing the dynamical properties of molecules. In particular, ab initio molecular dynamics methods have become standard tools in chemistry [1-4]. However, a quantum treatment of the nuclei becomes indispensable for the correct descriptions of zero-point vibrations and in cases where a tunneling effect is not negligible. There are a number of approaches that can be used to treat quantum effects approximately, such as the semiclassical [5-9] and semiquantal dynamics methods [10-14]. One of the notable works developed by Prezhdo et al. is the quantized Hamilton dynamics (QHD) approach derived from Heisenberg's equations of motion $[15,16]$. Nevertheless, it is too complicated to derive higher-order approximations due to the tedious manipulations required in decomposing the expectation values.

As an extension of the QHD, we have developed an approach called the quantal cumulant dynamics (QCD) method [17], where the cumulants are central variables in the formulation. In particular, we have derived a coupled equation of motion (EOM) for the position, momentum, and second-order cumulants of the product of the momentum and position fluctuation operators. The QCD approach has the following advantages over the QHD method: (i) A systematic 
construction of the higher-order equations of motion is possible. (ii) There are no errors in the energy and its gradient due to the truncation of the potential. (iii) There is no tedious derivation with respect to the decomposition scheme in the QHD method. We have shown that the method conserves the total energy exactly. However we demonstrated that numerical results obtained by a fourth-order Runge-Kutta method inherently suffers from accumulative error in the total energy for long time propagation.

Time-reversible integrators are used in the numerical simulation of Hamiltonian dynamics, because the trajectories and total energy evaluated by these methods remain accurate even at asymptotically long times [18-21]. Recent progress in the development of time-reversible integrators includes a multiple time scale algorithm and applications to non-Hamiltonian dynamics [22-25].

In the present letter, we develop a time-reversible integrator for the QCD method, where the key idea is to use a Liouville form of the time evolution operator. The equations of motion of the second-order quantal cumulant dynamics and the time-reversible integrator are given in Sec. II. Numerical tests for the time-reversible integrator are performed in Sec. III, and a summary is provided in Sec. IV.

\section{Theory}

\subsection{Quantal cumulant dynamics}

The Hamiltonian of a one-dimensional system is given by

$$
\hat{H}=\frac{1}{2} \hat{P}^{2}+V(\hat{Q})
$$


where the first term denotes the kinetic energy operator in a mass-weighted coordinate and the second term denotes the potential energy operator. These are functions of a momentum operator, $\hat{P}$, and a position operator, $\hat{Q}$. Here, we introduce a position shift operator defined as

$$
f(q+a)=\exp \left[a \frac{\partial}{\partial q}\right] f(q) .
$$

By using the shift operator, the potential energy operator can be rewritten as

$$
V(\hat{Q})=\left.\exp \left[(\hat{Q}-q) \frac{\partial}{\partial q^{\prime}}\right] V\left(q^{\prime}\right)\right|_{q^{\prime}=q}
$$

where $q=\langle\hat{Q}\rangle$ is the expectation value of $\hat{Q}$. By using the definition and a cumulant expansion technique for central moments [26,27], $(\hat{Q}-q)^{n}$, the potential energy can be approximated by a second-order cumulant as

$$
\langle V(\hat{Q})\rangle_{2}=\left.\exp \left[\frac{1}{2} \lambda_{2,0} \frac{\partial^{2}}{\partial q^{\prime 2}}\right] V\left(q^{\prime}\right)\right|_{q^{\prime}=q} \equiv \tilde{V}\left(q, \lambda_{2,0}\right)
$$

where $\lambda_{2,0}=\left\langle\delta \hat{Q}^{2}\right\rangle$ is a second-order cumulant of the central moment $\delta \hat{Q}=$ $\hat{Q}-q$. Hereafter we call the approximate expectation value of the potential energy operator, $\tilde{V}\left(q, \lambda_{2,0}\right)$, a quantal potential.

The kinetic energy is also expressed by the expectation value of the momentum operator, $p=\langle\hat{P}\rangle$, and that of its second-order cumulant, $\lambda_{0,2}=\left\langle\delta \hat{P}^{2}\right\rangle$. The second-order total energy is given by

$$
E_{2}=\frac{1}{2}\left(p^{2}+\lambda_{0,2}\right)+\tilde{V}\left(q, \lambda_{2,0}\right)
$$

To obtain a closed form for the coupled equations of motion for $q, p$, and the cumulant variables, we here introduce a further second-order cumulant of the product of the momentum and position fluctuation operators defined by 


$$
\lambda_{1,1}=\left\langle(\delta \hat{Q} \delta \hat{P})_{s}\right\rangle,
$$

where $s$ denotes a symmetric product; i.e. $(\delta \hat{A} \delta \hat{B})_{s}=1 / 2(\delta \hat{A} \delta \hat{B}+\delta \hat{B} \delta \hat{A})$. In the following we derive EOMs for all the variables defined above.

The Heisenberg EOM of an expectation value of an operator $\hat{A}$ is given by

$$
\mathrm{i}\left\langle\frac{\partial}{\partial t} \hat{A}\right\rangle=\left\langle[\hat{A}, \hat{H}]_{-}\right\rangle .
$$

where $[\cdots, \cdots]_{-}$is a commutator (in the unit of $\hbar$ ). Using this expression, the coupled EOMs in the second-order cumulant dynamics are given by

$$
\begin{aligned}
\dot{q} & =p, \\
\dot{p} & =-\tilde{V}^{(1)}\left(q, \lambda_{2,0}\right), \\
\dot{\lambda}_{2,0} & =2 \lambda_{1,1} \\
\dot{\lambda}_{0,2} & =-2 \lambda_{1,1} \tilde{V}^{(2)}\left(q, \lambda_{2,0}\right), \\
\dot{\lambda}_{1,1} & =\lambda_{0,2}-\lambda_{2,0} \tilde{V}^{(2)}\left(q, \lambda_{2,0}\right),
\end{aligned}
$$

where $\tilde{V}^{(n)}\left(q, \lambda_{2,0}\right)$ is the $n$-th derivative of the quantal potential with respect to $q$. These are central equations in the present letter. Here we should stress that the above EOMs conserve the total energy, though we adopt the secondorder approximation in the cumulant expansion. The initial conditions of the variables can be determined to be

$$
\begin{aligned}
q(0) & =q_{0}\left(\min E_{2}\left(q_{0}, p_{0}, \lambda_{2,0}=0, \lambda_{0,2}=0\right),\right. \\
p(0) & =p_{0}\left(\min E_{2}\left(q_{0}, p_{0}, \lambda_{2,0}=0, \lambda_{0,2}=0\right),\right. \\
\lambda_{2,0}(0) & =\eta, \\
\lambda_{0,2}(0) & =\xi, \\
\lambda_{1,1}(0) & =q(0) p(0) .
\end{aligned}
$$

In order to estimate the initial conditions, one needs to search for the point that satisfies the least quantal energy principle initially suggested by Tsue with the boundary condition $\eta \xi=1 / 4[13,14]$. 
In a previous work, we have established three ways to evaluate the quantal potential. In particular, we have demonstrated that the Morse potential has a closed form. The Morse potential and corresponding second-order quantal potential are given as

$$
\begin{aligned}
V_{\mathrm{M}}(q) & =\exp [-2 \alpha q]-2 \exp [-\alpha q] \\
\tilde{V}_{\mathrm{M}}\left(q, \lambda_{2,0}\right) & =\exp \left[-2 \alpha q+2 \alpha^{2} \lambda_{2,0}\right]-2 \exp \left[-\alpha q+\alpha^{2} \lambda_{2,0} / 2\right] .
\end{aligned}
$$

Once the analytic form of the quantal potential is obtained, its higher-order derivatives are easily evaluated as

$$
\begin{aligned}
& \tilde{V}_{\mathrm{M}}^{(1)}\left(q, \lambda_{2,0}\right)=-2 \alpha \exp \left[-2 \alpha q+2 \alpha^{2} \lambda_{2,0}\right]-2 \alpha \exp \left[-\alpha q+\alpha^{2} \lambda_{2,0} / 2\right] \\
& \tilde{V}_{\mathrm{M}}^{(2)}\left(q, \lambda_{2,0}\right)=4 \alpha^{2} \exp \left[-2 \alpha q+2 \alpha^{2} \lambda_{2,0}\right]-2 \alpha^{2} \exp \left[-\alpha q+\alpha^{2} \lambda_{2,0} / 2\right]
\end{aligned}
$$

The potential and their derivatives can be calculated at any $q$ and $\lambda_{2,0}$ as in a standard classical dynamics simulation.

\subsection{Time-reversible integrator for $Q C D$}

An efficient time-reversible integrator was initially proposed by Yoshida for Hamiltonian dynamics, and is well-known as higher-order symplectic integrators [18-19]. Independently Suzuki established the basics of a Lie-algebraic construction of a decomposed time evolution operator [20], which can be applied not only to the classical Hamiltonian dynamics, but also to quantum dynamics. In the following, we give a time-reversible integrator for the QCD derived with a consistent manner.

The time evolution of the above variables is described in terms of a propagator from $t=0$ to $t=\delta t$ as

$$
\boldsymbol{a}(\delta t)=\exp (\mathrm{i} L \delta t) \boldsymbol{a}(0)
$$


where $\boldsymbol{a}$ is a vector that consists of the above five variables defined above and $L$ is the Liouville operator of the QCD. A variety of integrators exist, which differ from one another in the way of approximating the time evolution operator. According to the EOMs, the Liouville operator in a vector form is expressed as

$$
\mathrm{i} L=\boldsymbol{X} \cdot \mathrm{d} \boldsymbol{Z}^{\dagger}=\sum_{m} X_{m} \mathrm{~d} Z_{m} \quad\left(m=q, p, \lambda_{2,0}, \lambda_{1,1}, \text { and } \lambda_{0,2}\right),
$$

where

$$
\boldsymbol{X}^{\dagger}=\left(\begin{array}{c}
p \\
-\tilde{V}^{(0)}\left(q, \lambda_{2,0}\right) \\
2 \lambda_{1,1} \\
\lambda_{0,2}-\lambda_{2,0} \tilde{V}^{(2)}\left(q, \lambda_{2,0}\right) \\
-2 \lambda_{1,1} \tilde{V}^{(2)}\left(q, \lambda_{2,0}\right)
\end{array}\right),
$$

and

$$
\mathrm{d} \boldsymbol{Z}^{\dagger}=\left(\begin{array}{c}
\frac{\partial}{\partial q} \\
\frac{\partial}{\partial p} \\
\frac{\partial}{\partial \lambda_{2,0}} \\
\frac{\partial}{\partial \lambda_{1,1}} \\
\frac{\partial}{\partial \lambda_{0,2}}
\end{array}\right) .
$$

The time-reversible integrators can be derived in the following manner: The 
Liouville operator is decomposed into three non-commutative operators $L_{1}$, $L_{2}$, and $L_{3}$ as

$$
\mathrm{i} L=\mathrm{i}\left(L_{1}+L_{2}+L_{3}\right) \equiv \sum_{m_{1}} X_{m_{1}} \mathrm{~d} Z_{m_{1}}+\sum_{m_{2}} X_{m_{2}} \mathrm{~d} Z_{m_{2}}+\sum_{m_{3}} X_{m_{3}} \mathrm{~d} Z_{m_{3}}
$$

Since there are several ways to decompose the Liouvillian, we here adopt three of them so as to require the least number of force evaluations. The definitions of $L_{k}$ are listed in Table 1. By decomposing the time evolution operator into three parts, one can obtain a sequential time evolution operator as

$$
\exp [\mathrm{i} L \delta t] \approx \prod_{j=1}^{k}\left[\exp \left(\mathrm{i} c_{j, 1} L_{1} \delta t\right) \exp \left(\mathrm{i} c_{j, 2} L_{2} \delta t\right) \exp \left(\mathrm{i} c_{j, 3} L_{3} \delta t\right)\right]
$$

where a set of real coefficients $c_{j, k}(j=1,2$, and 3$)$ is determined by comparing the Taylor expansion of the partitioned-time evolution operator with that of the whole time evolution operator. For example, a second-order approximation to the time evolution operator is given by

$$
\begin{aligned}
\exp [\mathrm{i} L \delta t] & \approx \exp \left[\mathrm{i} L_{3} \delta t / 2\right] \exp \left[\mathrm{i} L_{2} \delta t / 2\right] \exp \left[\mathrm{i} L_{1} \delta t\right] \exp \left[\mathrm{i} L_{2} \delta t / 2\right] \exp \left[\mathrm{i} L_{3} \delta t / 2\right] \\
& \equiv S_{2}(d t) .
\end{aligned}
$$

This decomposition is correct to third-order with respect to the time step, $\delta t$. The fourth-order integrator is constructed from a product of second-order evolution operators with different time steps as

$$
S_{4}(\delta t)=S_{2}(s \delta t) S_{2}(s \delta t) S_{2}((1-4 s) \delta t) S_{2}(s \delta t) S_{2}(s \delta t)
$$

where $s=1 /(4-\sqrt[3]{4})$. In this letter, we adopt the above $S_{2}$ and $S_{4}$ for actual calculations. 


\section{Results and discussion}

Numerical simulations of the QCD were performed with the Runge-Kutta integrator and the time-reversible integrators devised here. The parameter of the Morse potential in our simulations was $\alpha=0.1$. The initial conditions used in the simulation were $p=q=\lambda_{1,1}=0, \lambda_{2,0}=3.33885$, and $\lambda_{0,2}=1 / 4 \lambda_{2,0}$. According to the exact quantum result, the total energy of the ground state is $E_{\text {exact }}=-0.930539$, whereas the initial energy obtained by means of the present scheme is $E_{2}=-0.927174$.

The accuracy of the QCD was evaluated in terms of the conservation of the total energy. Figures 1-(a) and 1-(b) depict time series of the maximum error of the total energy against the number of gradient and Hessian updates per 1000 unit time, $N$. Here the number of updates and the error are defined as

$$
\begin{aligned}
N & =1000 \quad n_{x} / \delta t, \\
\text { error } & =\max \log _{10}\left(\left|E_{2}(t)-E_{2}(0)\right|\right),
\end{aligned}
$$

where $n_{x}$ is the number of the updates for a unit time step, $\delta t$, required in the algorithm $x$.

Since one of the most time consuming steps in a molecular dynamics simulation is the update of the gradient and Hessian, it is instructive to measure the error against $N$ rather than $\delta t$ in order to estimate the efficiency in actual calculations. It is necessary to update the gradient and Hessian of the quantal potential once for the second-order time-reversible integrator, twice for the second-order Runge-Kutta, five times for the fourth-order time-reversible integrator, and four times for the forth-order Runge-Kutta. 
Comparing the time-reversible integrators, Scheme A is the least accurate, and Scheme $\mathrm{C}$ is the most accurate of the three schemes. Since the EOMs of $\lambda_{0,2}$ and $\lambda_{1,1}$ depend mutually on each other, the error becomes critically large with Scheme A. In particular, the error in the fourth-order method using Scheme A is very similar to that in the second-order method (not shown in the figure). It is thus concluded that the partition of $p-\lambda_{0,2}$ and $\lambda_{1,1}$ cannot be disregarded. Scheme $\mathrm{C}$ is more accurate than that of $\mathrm{B}$ by one order of magnitude, and Schemes $\mathrm{B}$ and $\mathrm{C}$ differ in the order of the time evolution operators of $p-\lambda_{0,2}$ and $\lambda_{1,1}$. This fact tells us that the equations of motion of the QCD are sensitive to the order in the time-reversible integrator. Judging from the figures, the time-reversible integrator (Scheme C) is always superior to the Runge-Kutta method at the same order.

To evaluate the applicability of the fourth-order methods to long-time simulation, we adopt a relatively large time step, $\delta t=5$. Time series of the short time averaged-error defined as

$$
\operatorname{error}_{\mathrm{av}}(t)=\frac{1}{N_{\mathrm{av}}} \sum_{i=0}^{N_{\mathrm{av}}} \log \quad\left(\left|E_{2}(t+i \delta t)-E_{2}(0)\right|\right)
$$

are depicted in Fig. 2. Scheme A no longer conserves the total energy. Both Schemes B and C are so robust that the averaged error is almost constant. The errors are estimated to be $10^{-4.6}$ for Scheme B and $10^{-5.5}$ for Scheme C. From Fig. 2, when we use the second-order algorithm, the latter error is equivalent to using a time step, $\delta t=0.5$. Thus the fourth-order method enables one to adopt a time step ten times larger in this case. Because the second- and fourth-order algorithms require one and five force updates, respectively, the fourth-order method is twice as fast as the second-order method overall. The error by using the Runge-Kutta method reaches a plateau after $T=10^{4}$, 
where the error becomes approximately $10^{-2.6}$. Nevertheless the trajectories generated by the Runge-Kutta are divergent as shown in Fig. 3. On the other hand, the trajectory by Scheme $\mathrm{C}$ is almost the same as that obtained by Scheme $\mathrm{C}$ with a short time step $\delta t=0.5$, where the maximum error in the total energy is $10^{-9.3}$. Therefore, we conclude here that the fourth-order time-reversible integrator with Scheme $\mathrm{C}$ is robust and stable for long time simulation. Finally, we note that such integrators naturally depend on the system of interest, and we should explore the application of the method to larger systems, such as the vibration of polyatomic molecules.

In order to recognize the difference between Scheme B and C, we here show a short-time propagation using the second-order time-reversible integrator with Scheme C as

$$
\left(\begin{array}{c}
q(\delta t) \\
p(\delta t) \\
\lambda_{2,0}(\delta t) \\
\lambda_{1,1}(\delta t) \\
\lambda_{0,2}(\delta t)
\end{array}\right)=\left(\begin{array}{l}
q(0)+p(\delta t / 2) \delta t \\
p(0)-\left[\tilde{V}^{(1)}(0)+\tilde{V}^{(1)}(\delta t)\right] \delta t / 2 \\
\lambda_{2,0}(0)+2 \lambda_{1,1}(\delta t / 2) \delta t \\
\lambda_{1,1}(0)+\left[\lambda_{0,2}(0)+\lambda_{0,2}(\delta t)-\lambda_{2,0}(0) \tilde{V}^{(2)}(0)-\lambda_{2,0}(\delta t) \tilde{V}^{(2)}(\delta t)\right] \delta t / 2 \\
\lambda_{0,2}(0)-\lambda_{1,1}(\delta t / 2)\left[\tilde{V}^{(2)}(0)+\tilde{V}^{(2)}(\delta t)\right] \delta t
\end{array}\right),
$$

where $\tilde{V}(0)$ and $\tilde{V}(\delta t)$ are the quantal potential at $t=0$ and $t=\delta t$. It is found that the fractional time of $\lambda_{1,1}(\delta t / 2)$ in $\lambda_{2,0}(\delta t)$ and $\lambda_{0,2}(\delta t)$ is the same. It should be stressed here that this does not hold true for Scheme B. Figures 1 and 2 indicate that there exists a conserving quantity which is invariant under the time propagation. The error in the total energy comes from the difference 
between the total energy and the conserving quantity. Since the total energy depends on four variables except for $\lambda_{1,1}$, the accordance of fractional time in $\lambda_{2,0}(\delta t)$ and $\lambda_{0,2}(\delta t)$ is desirable for the conservation of energy.

Since the time-reversible integrator is robust for long-time step simulation, the present method is applicable to systems whose motions have different time scales such as fast electronic and slow nuclear motions. This method is also applicable to non-Born-Oppenheimer (NBO) treatments for molecular calculations, which are a challenge in this area of research, and have been developed by one of the present authors and by other groups [28-35].

\section{Concluding remarks}

We have constructed a time-reversible integrator for the QCD method. The key point of the work is the use of a Liouville form for the time evolution operator. In particular, we have developed three schemes $\mathrm{A}, \mathrm{B}$, and $\mathrm{C}$ listed in Table 1 by partitioning the Liouvillian and arranging the decomposed time evolution operators. The time-reversible integrator except for Scheme A is superior to the conventional Runge-Kutta method for long time propagation. We have numerically elucidated that Scheme $\mathrm{C}$ is more reliable than the other schemes both for second- and fourth-order approximations. This indicates that the EOM of QCD is sensitive not only to the partition, but also to the order in the decomposed integrator. We have suggested that the accordance of fractional time in $\lambda_{2,0}$ and $\lambda_{0,2}$ is desirable for the conservation of energy, because these variables contribute to the total energy.

Since the time-reversible integrator enables adoption of relatively long time 
steps, Scheme C with the fourth-order approximation requires the least number of updates, although the fourth-order time-reversible integrator requires five force updates per time step propagation. In particular, one can choose $\delta t=5$ in Scheme $\mathrm{C}$ with a maximum error of $10^{-5.5}$, where, in contrast, the Runge-Kutta method generates an incorrect divergent trajectory.

\section{Acknowledgment}

This research was supported by the Core Research for Evolutional Science and Technology (CREST) Program "High Performance Computing for Multi-Scale and Multi-Physics Phenomena" of the Japan Science and Technology Agency (JST). YS acknowledges M.A. Watson for his helpful advice. 


\section{References}

[1] R. Car, M. Parrinello, Phys. Rev. Lett. 55 (1985) 2471.

[2] D. Marx, J. Hutter, in J. Grotendorst(Ed.), NIC Series Vol. 1 Modern methods and algorithms of Quantum Chemistry, Proceedings, Forschungszentrum Jülich, $301-449,2000$.

[3] Y. Shigeta, K. Takatsuka, J. Chem. Phys. 123 (2005) 131101.

[4] A. Furuhama, K. Hirao, M. Dupuis, J. Chem. Phys. 124 (2006) 164310.

[5] J.C. Tully, Ann. Rev. Phys. Chem. 51 (2000) 153.

[6] W.H. Miller, J. Phys. Chem. A 105 (2001) 2942.

[7] G.A. Fiete, E.J. Heller, Rev. Mod. Phys. 75 (2003) 933.

[8] J.L. Gao, D.G. Truhlar, Ann. Rev. Phys. Chem. 53 (2002) 467.

[9] I. Burghardt, J. Chem. Phys. 122 (2005) 094103.

[10] K. Ando, J. Chem. Phys. 125 (2006) 014104.

[11] R. Jackiw, A. Kerman, Phys. Lett. A. 71 (1979) 158.

[12] K. Cooper, S.-Y. Pi, P.N. Stancioff, Phys. Rev. D 34 (1986) 3831.

[13] Y. Tsue and Y. Fujiwara, Prog. Theoret. Phys. 86 (1991) 443.

[14] Y. Tsue, Prog. Theoret. Phys. 88 (1992) 911.

[15] O.V. Prezhdo, Theoret. Chem. Acc. 116 (2006) 206 and references cited therein.

[16] H. Miyachi, Y. Shigeta, K. Hirao, Chem. Phys. Lett. 432 (2006) 585.

[17] Y. Shigeta, H. Miyachi, K. Hirao, J. Chem. Phys. 125 (2006) 244102.

[18] H. Yoshida, Phys. Lett. A 150 (1990) 262. 
[19] H. Yoshida, Celest. Mech. A56 (1993) 27.

[20] M. Suzuki, Phys. Lett. A 146 (1990) 319.

[21] B. Leimkuhler, S. Reich, Simulating Hamiltonian Dynamics, Cambridge Monograph on Applied and Computational Mathematics, Vol. 14, Cambridge University Press, Cambridge, UK, 2004.

[22] S. Nosé, Prog. Theo. Phys. Suppl. 103 (1991) 46.

[23] W.G. Hoover, Phys. Rev. A31 (1985) 1895.

[24] M. E. Tuckerman, M. Parrinello, J. Chem. Phys. 101 (1994) 1302.

[25] G. J. Martyna, M. E. Tuckerman, D. J. Tobias, M. L. Klein, Mol. Phys. 87 (1996) 1117.

[26] J.E. Mayer, J. Chem. Phys. 5 (1937) 67.

[27] R. Kubo, J. Phys. Soc. Jpn. 17 (1962) 1100.

[28] Y. Shigeta, K. Kodama, H. Nagao, H. Kawabe, K. Nishikawa, Int. J. Quantum Chem. 69 (1998) 629.

[29] Y. Shigeta, H. Nagao, K. Nishikawa, K. Yamaguchi, Int. J. Quantum Chem. 70 (1998) 659.

[30] Y. Shigeta, H. Nagao, K. Nishikawa, K. Yamaguchi, J. Chem. Phys. 111 (1999) 6171.

[31] M. Tachikawa, K. Mori, H. Nakai, K. Iguchi, Chem. Phys. Lett. 290 (1998) 437.

[32] S.P. Webb, T. Iordanov, S. Hammes-Schiffer, J. Chem. Phys. 117 (2002) 4106.

[33] K. Sodeyama, K. Miyamoto, H. Nakai, Chem. Phys. Lett. 421 (2006) 72.

[34] C. Swalina, M.V. Pak, A. Chakraborty, S. Hammes-Schiffer, J. Phys. Chem. A $110(2006) 9983$. 
[35] T. Ishimoto, M. Tachikawa, U. Nagashima, Int. J. Quantum Chem. 106 (2006) 1465. 


\section{Tables}

Table 1

Terms in decomposed Liouvillians

\begin{tabular}{ccc}
\hline \hline$L_{1}$ & $L_{2}$ & $L_{3}$ \\
& & \\
$m_{1}$ & $m_{2}$ & $m_{3}$ \\
\hline
\end{tabular}

Scheme A $\quad q, \lambda_{2,0} \quad p, \lambda_{1,1}, \lambda_{0,2}$

Scheme B $\quad q, \lambda_{2,0} \quad \lambda_{1,1} \quad p, \lambda_{0,2}$

Scheme C $\quad q, \lambda_{2,0} \quad p, \lambda_{0,2} \quad \lambda_{1,1}$ 


\section{Figure captions}

\section{Fig. 1.}

Maximum error vs. the number of updates for the second-order (a) RungeKutta (solid circle), Schemes A (solid square), B (diamond), and C (X) and those for the forth-order (b).

\section{Fig. 2 .}

Time series of the averaged absolute relative error obtained using the fourthorder (i) Runge-Kutta (solid circle), (ii) Scheme B (solid square), (iii) C (solid triangle). The time step is $\delta t=5$ and the number of sampling steps is $N_{\mathrm{av}}=$ 50.

Fig. 3.

Phase space structure obtained using Scheme C and Runge-Kutta. The orbit of Scheme $\mathrm{C}$ is in reasonable agreement with that obtained by setting a shorter time step, $\delta t=0.5$, whereas the Runge-Kutta method gives an incorrect divergent trajectory though the energy is at least conserved (see Fig. 2). 
(a)

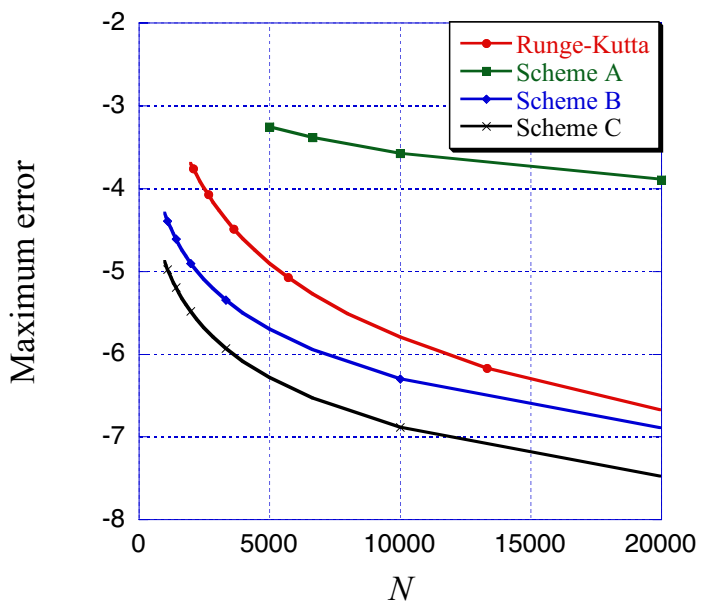

(b)

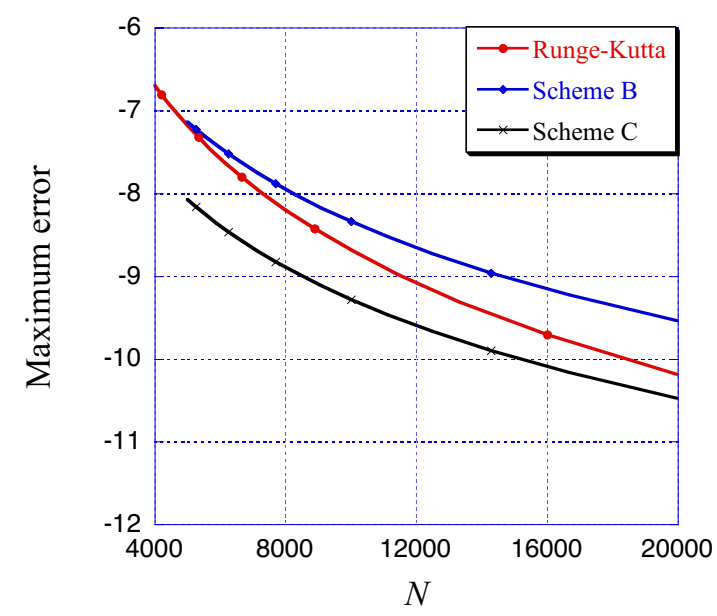

Figure 1. Y. Shigeta et al. 


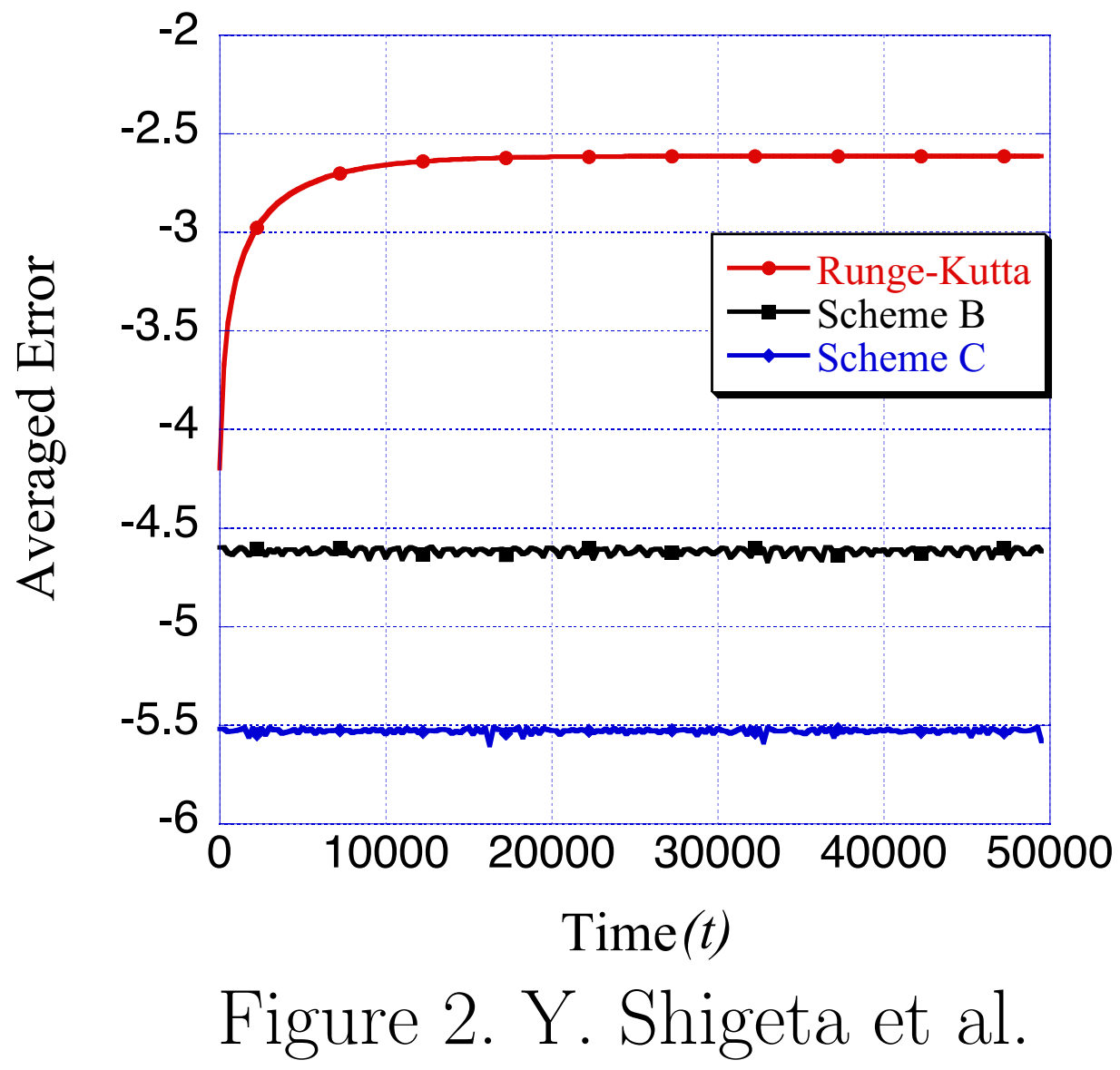




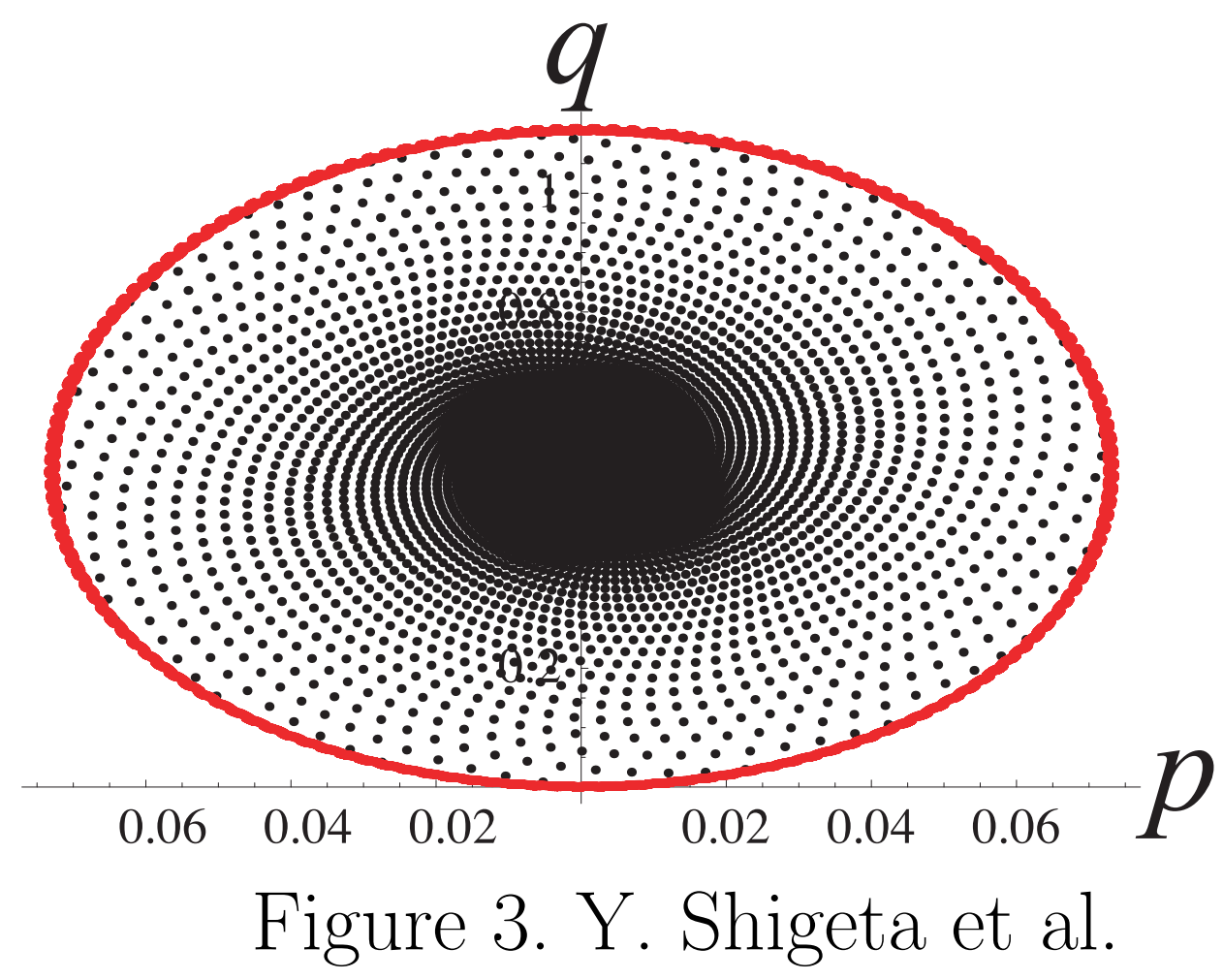

